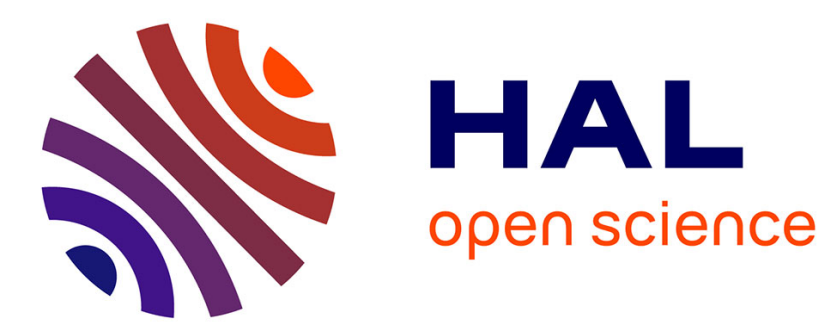

\title{
Molecular predictors of efficacy of adjuvant weekly paclitaxel in early breast cancer
}

Miguel Martín, Álvaro Rodríguez-Lescure, Amparo Ruiz, Emilio Alba, Lourdes Calvo, Manuel Ruiz-Borrego, Ana Santaballa, César A. Rodríguez, Carmen Crespo, Mar Abad, et al.

\section{To cite this version:}

Miguel Martín, Álvaro Rodríguez-Lescure, Amparo Ruiz, Emilio Alba, Lourdes Calvo, et al.. Molecular predictors of efficacy of adjuvant weekly paclitaxel in early breast cancer. Breast Cancer Research and Treatment, 2009, 123 (1), pp.149-157. 10.1007/s10549-009-0663-z . hal-00535419

\section{HAL Id: hal-00535419 https://hal.science/hal-00535419}

Submitted on 11 Nov 2010

HAL is a multi-disciplinary open access archive for the deposit and dissemination of scientific research documents, whether they are published or not. The documents may come from teaching and research institutions in France or abroad, or from public or private research centers.
L'archive ouverte pluridisciplinaire HAL, est destinée au dépôt et à la diffusion de documents scientifiques de niveau recherche, publiés ou non, émanant des établissements d'enseignement et de recherche français ou étrangers, des laboratoires publics ou privés. 


\title{
Molecular predictors of efficacy of adjuvant weekly paclitaxel in early breast cancer
}

\author{
Miguel Martín • Álvaro Rodríguez-Lescure • Amparo Ruiz • Emilio Alba · Lourdes Calvo • \\ Manuel Ruiz-Borrego $\cdot$ Ana Santaballa $\cdot$ César A. Rodríguez $\cdot$ Carmen Crespo \\ Mar Abad · Severina Domínguez · Jesús Florián · Cristina Llorca • Miguel Méndez • \\ María Godes · Ricardo Cubedo $\cdot$ Adolfo Murias · Norberto Batista $\cdot$ María José García • \\ Rosalía Caballero · Enrique de Álava
}

Received: 25 August 2009/Accepted: 21 November 2009/Published online: 27 December 2009

(C) Springer Science+Business Media, LLC. 2009

\begin{abstract}
Treatment with fluororacil, epirubicin, and cyclophosphamide followed by weekly paclitaxel (FEC-P) yielded superior disease-free survival than FEC in the adjuvant breast cancer trial GEICAM 9906. We evaluate molecular subtypes predictive of prognosis and paclitaxel response in this trial. Two molecular subtype classifications based on conventional immunohistochemical and fluorescent in situ hybridization determinations were used: \#1: Four groups segregated according to the combination of hormone receptor (HR) and HER2 status; \#2: Intrinsic subtype classification (Triple Negative (TN), HER2, Luminal B and Luminal A). Results: Both subtype classifications yielded prognostic and predictive information. HR
\end{abstract}

This study is conducted on behalf of the GEICAM 9906 study investigators.

M. Martín ( $₫)$

Servicio de Oncología Médica, Departmento de Oncología,

Hospital General Universitario Gregorio Marañón,

c/Maiquez 7, 28009 Madrid, Spain

e-mail: mmartin@geicam.org

Á. Rodríguez-Lescure

Department of Medical Oncology, Hospital Universitario

de Elche, Elche, Spain

A. Ruiz

Department of Medical Oncology, Instituto Valenciano

de Oncología, Valencia, Spain

E. Alba

Department of Medical Oncology, Hospital Virgen

de la Victoria, Málaga, Spain

L. Calvo

Department of Medical Oncology, Complexo Hospitalario

Universitario A Coruña, A Coruña, Spain
+/HER2 - patients (and Luminal A patients) had a significantly better outcome than the other subgroups of patients. The superiority of FEC-P over FEC was clearly more marked in HR-/HER2- patients (TN patients), particularly in the subset with basal phenotype (TN and either EGFR+ or cytokeratins 5/6+). The Luminal A subtype also achieved a significant benefit with FEC-P. The molecular-defined subgroup of TN was clearly predictive of better response to treatment with FEC-P. Luminal A patients had the best prognosis and also have a better outcome with weekly paclitaxel.

Keywords Breast cancer - Adjuvant paclitaxel . Molecular predictors of efficacy

M. Ruiz-Borrego

Department of Medical Oncology, Hospital Vírgen

del Rocío, Sevilla, Spain

A. Santaballa

Department of Medical Oncology, Hospital La Fe,

Valencia, Spain

C. A. Rodríguez

Department of Medical Oncology, Hospital Universitario

de Salamanca, Salamanca, Spain

C. Crespo

Department of Medical Oncology, Hospital Ramón y Cajal, Madrid, Spain

M. Abad

Department of Cellular Biology and Pathology, Facultad de Medicina, Universidad de Salamanca, Salamanca, Spain 


\section{Introduction}

Several adjuvant therapy trials comparing anthracycline versus taxane containing regimens have shown an absolute improvement in disease-free survival (DFS) of 4-7\% with taxanes [1-6]. Many attempts have been made to identify the molecular peculiarities of the tumors of the patients who may obtain the greater benefit from taxanes [7]. A study by the CALGB group has suggested that patients who obtain the maximum benefit from paclitaxel are those whose tumors overexpress HER2 [8]. Similarly, the classification into four intrinsic subtypes, as defined by Perou et al. [9], (i.e., Luminal A, Luminal B, HER2 and basallike), has been shown recently to be of prognostic and predictive value. A simple "immunopanel", based on standard immunohistochemical (IHC) measurements and fluorescent in situ hybridization (FISH), has been used recently to segregate the patients from the BCIRG 001 trial into these same subtypes [10]. We have previously reported the 5-year results of GEICAM 9906, an adjuvant trial in node positive breast cancer in which four cycles of FEC followed by eight cycles of weekly paclitaxel yielded a superior 5-year DFS, but not 5-year overall survival (OS), compared to six cycles of FEC [11].

We report, here, an analysis of the prognostic and predictive value of paclitaxel efficacy of two classifications of tumors based on conventional pathological parameters.

\section{Methods}

GEICAM 9906 was an adjuvant multi-centered randomized phase III study of six cycles of FEC (control arm)

\author{
S. Domínguez \\ Department of Medical Oncology, Hospital Txagorritxu, \\ Vitoria, Spain \\ J. Florián \\ Department of Medical Oncology, Hospital Comarcal \\ Barbastro, Barbastro, Spain \\ C. Llorca \\ Department of Medical Oncology, Hospital de Elda, Elda, \\ Spain \\ M. Méndez \\ Department of Medical Oncology, Hospital General de \\ Móstoles, Móstoles, Spain \\ M. Godes \\ Department of Medical Oncology, Hospital General \\ Universitario de Valencia, Valencia, Spain \\ R. Cubedo \\ Department of Medical Oncology, Hospital Puerta \\ de Hierro, Madrid, Spain
}

versus four cycles of FEC followed by eight cycles of weekly paclitaxel (experimental arm) for node-positive breast cancer. The characteristics of the patients have been previously reported [11].

The primary endpoint of the trial was 5-year DFS, according to the definition of Invasive Disease Free Survival (IDFS) in the Standardized Definitions for Efficacy and End Points in Adjuvant Breast Cancer Trials (STEEP) System [12]. Secondary endpoints were: (1) overall survival, defined as the time from randomization until death from any cause; (2) prognostic and predictive value of the molecular markers (HR status and HER2 status); (3) safety.

The study was approved by the institutional review boards of all participating hospitals and by the Spanish Government Health Authorities and was registered at www.clinicaltrials. gov (identifier code: NCT00129922). All patients signed an informed consent form before being enrolled in the trial. Patients were also requested to sign a specific informed consent for molecular analyses of their tumors.

Immunohistochemical and fluorescent in situ hybridization analysis of tumors

Paraffin-embedded tumor samples from the primary tumors of the patients who consented were processed centrally. Tumor tissue was analyzed in tissue microarrays of three separate cores (each of $0.6 \mathrm{~mm}$ in diameter) from each tumor sample. The hormone receptor status of tumors was evaluated by IHC analysis for progesterone receptor (PR) (anti-progesterone receptor antibody clone PgR636, dilution 1:50, product M3569, DAKO, Carpinteria, CA, USA) and estrogen receptor (ER) (anti-estrogen receptor antibody clone 1D5, dilution 1:35, product M7047, DAKO)

\author{
A. Murias \\ Department of Medical Oncology, Hospital Universitario \\ Insular, Las Palmas, Spain \\ N. Batista \\ Department of Medical Oncology, Hospital Universitario De \\ Canarias, Tenerife, Spain \\ M. J. García \\ Department of Medical Oncology, Hospital General de Ciudad \\ Real, Ciudad Real, Spain \\ R. Caballero \\ GEICAM, Madrid, Spain \\ E. de Álava $(\square)$ \\ Molecular Pathology, Centro de Investigación del Cancer- \\ IBMCC, Universidad de Salamanca- CSIC, Salamanca, Spain
}


using the DAB Map system (Ventana Medical Systems, Tucson, AZ, USA). Staining was initially scored according to the Allred method [13]. However, to compare the results of our study with those of BCIRG001 [10], we reclassified our samples according to ER+ or PR+ if staining was present in $\geq 1 \%$ of tumor nuclei. Tumors were considered $\mathrm{HR}+$ if they were $\mathrm{ER}+$ or $\mathrm{PR}+$.

HER2 status was evaluated by FISH (HER2 FISH pharmDx kit, product K5331,DAKO, Glostrup, Denmark) according to recent recommendations [14]. A positive result was defined as a HER2 gene to chromosome 17 ratio of $>2$. A semiquantitative scoring system was employed to evaluate the amount of cells expressing all other markers (Ki67, cytokeratins 5/6 and EGFR). The scoring was $0=$ negative; $\mathrm{E}=$ rare cells, $<10 \% ; 1+=10 \%$ up to $1 / 3$ of cells; $2+=$ between one-third and two-thirds of cells; $3+=$ more than two-thirds of cells.

Results for Ki67 were recoded as Ki67high (scores 1+, 2+, 3+), and Ki67low (scores 0, E). Cytokeratins 5/6 and epidermal growth factor were considered positive when any staining was present (scores $\mathrm{E}, 1+, 2+, 3+$ ).

To analyze the prognostic and predictive value of molecular subgroups, two classification systems based on the pathology parameters defined above (HR status, HER status, plus or minus Ki67) were used

(1) HR/HER2 classification: four groups were segregated according to the combination of HR and HER2 status (i.e., HR+/HER2+, HR+/HER2-, HR-/HER2+, and HR-/HER2-).

(2) Intrinsic molecular subtypes: this classification is based in the concept that a simple "immunopanel" of ER, PR, HER2 and Ki67 can serve as a proxy for a more complex genetic subtyping [10]. Patients were classified as: (1) Triple Negative (TN) (ER-, PR-, HER2-); (2) HER2 (ER-, PR-, HER2+); (3) Luminal B (ER+ and/or PR+ and either HER2+ and/or Ki67high); (4) Luminal A (ER+ and/or PR+ and not HER2+ or Ki67high). As an additional exploratory analysis, we assessed the outcome of the subset of patients showing basal phenotype (TN and EGFR positive and/or cytokeratins $5 / 6+$ ), since this phenotype has been shown to have superior prognostic value to the TN phenotype [15].

The results are presented in accordance with reporting recommendations for tumor marker prognostic studies criteria [16].

\section{Statistical analyses}

Survival variables (i.e., relapse and death) were analyzed with the Kaplan-Meier method [17]. Since patients belonging to some subgroups (i.e., those with hormone receptor-positive tumors) can have late recurrences, we made a 7-year update of the results of the trial in order to have more reliable recurrence data. The log-rank test was used to compare time-to-event data between treatment arms, or between subgroups. Hazard ratios and $95 \%$ confidence intervals $(95 \% \mathrm{CI})$ were obtained from the Cox's proportional hazards models. The hypotheses tests were two-sided, and the values for alpha and beta accepted as being statistically significantly were 0.05 and 0.2 , respectively.

The relative efficacy of FEC-P versus FEC in terms of DFS was analyzed in each of the previously described molecular subgroups. We performed a Cox proportional hazards model to evaluate the effect of chemotherapy on DFS in these subgroups. To rule out any confounding effects of other variables we included the age, menopausal status, tumor size, lymph node status, and hormonal therapy in the model.

\section{Results}

At a median follow-up of 7 years, a total of 364 DFS events have been recorded (205 in the FEC arm and 159 in the FEC$\mathrm{P}$ arm (hazards ratio: 0.75 ; 95\% CI: $0.61-0.93 ; P=0.007$ ). The 7-year DFS was significantly superior in the FEC-P arm (75\% vs. $68 \% ; P=0.007$ log-rank test). There have been 217 deaths (125 in the FEC arm and 92 in the FEC-P arm (hazards ratio: $0.74 ; 95 \%$ CI: $0.56-0.96 ; P=0.026$ ). The 7year OS was also significantly superior in the FEC-P arm (84 vs. $79 \% ; P=0.025$ log-rank test) (Fig. 1a, b).

\section{Molecular subtype analysis}

Tumor samples for evaluation in a central laboratory were available from $928(74.5 \%)$ of the 1,246 patients in the trial. The demographic and prognostic features, as well as 7-year DFS and OS, of patients whose tumor samples had been centrally tested were similar to those that had not been centrally tested (data not shown).

Figure 2a and $\mathrm{b}$ show Kaplan-Meier analyses for DFS in the two molecular classifications. In the HR/HER2 classification (Fig. 2a), HR+/HER2 - patients had a significantly better DFS than the rest of the groups $(P<0.0001)$. In the intrinsic subtype classification, Luminal A patients had the best DFS outcomes $(P<.0001$ for Luminal A vs. TN + HER2; and $P<0.0001$ for Luminal A vs. Luminal B). TN and HER2 had the worst outcomes. Luminal B patients had an intermediate outcome, although the difference with TN + HER2 was not statistically significant (HR for DFS: $0.85 ; 95 \%$ CI: $0.65-1.11 ; P=0.227)$. 

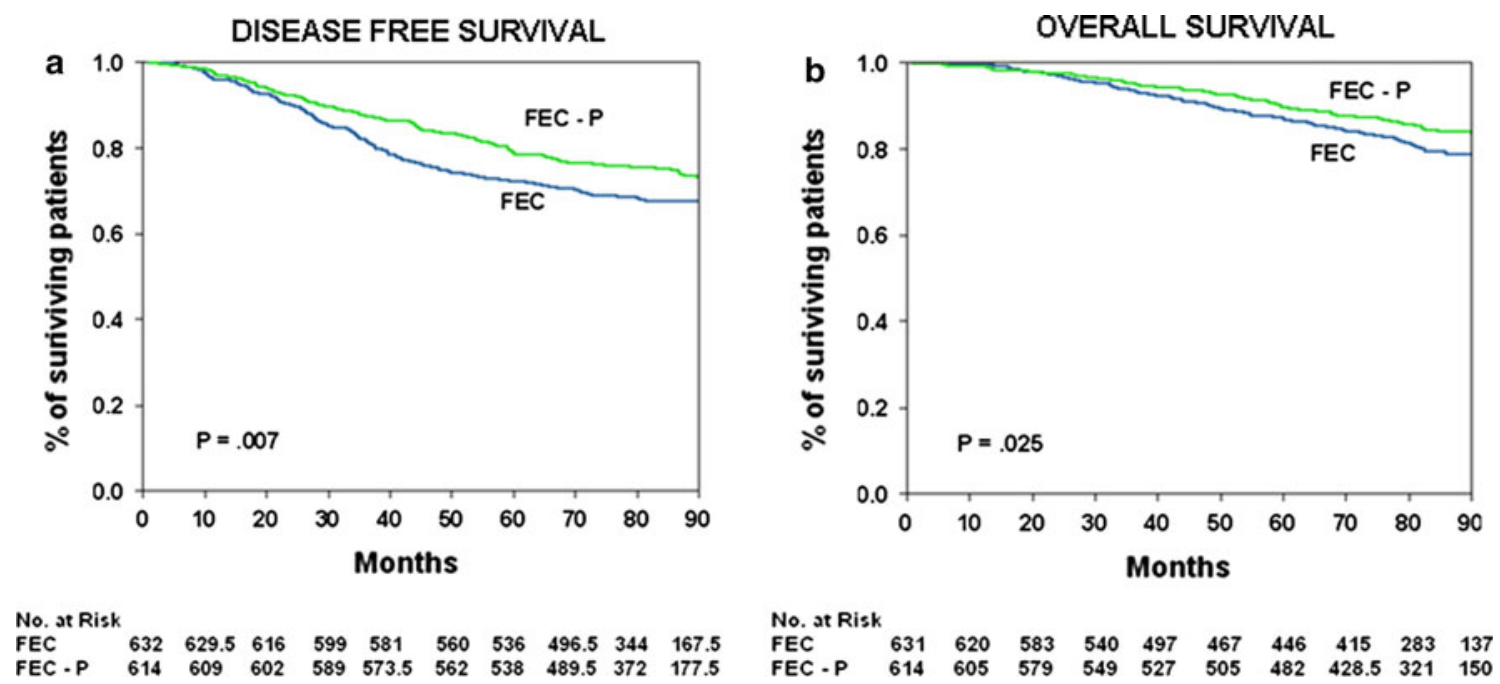

Fig. 1 7-year disease-free survival (DFS) (a), and overall survival (b) of the whole population according to chemotherapy arm

a

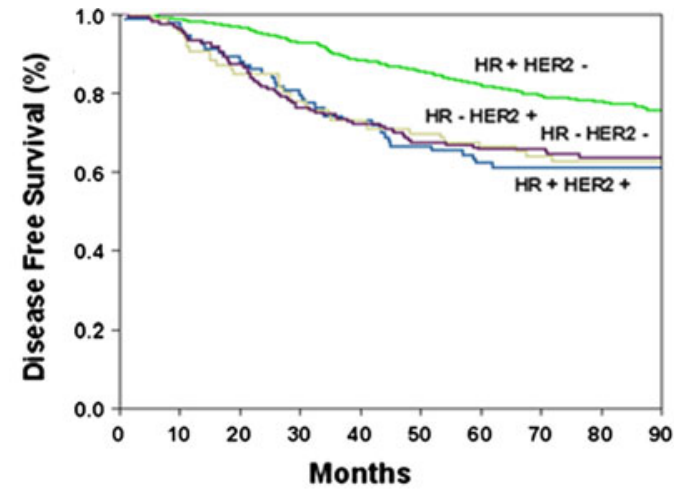

No. at Risk

$\begin{array}{lcccccccccc}\text { HR + HER2 + } & 93 & 91 & 83 & 75 & 67 & 61 & 58 & 54 & 39 & 17.5 \\ \text { HR + HER2. } & 549 & 542 & 531 & 509 & 484.5 & 468 & 445.5 & 400.5 & 279.5 & 125.5\end{array}$ $\begin{array}{lcccccccccc}\text { HR + HER2 - } & 549 & 542 & 531 & 509 & 484.5 & 468 & 445.5 & 400.5 & 279.5 & 125.5 \\ \text { HR. HER2 + } & 86 & 84 & 73 & 67 & 63 & 60 & 58 & 52 & 39 & 13.5 \\ \text { HR. HER2. } & 169 & 165 & 148 & 131 & 122 & 114 & 110 & 105 & 79.5 & 47\end{array}$
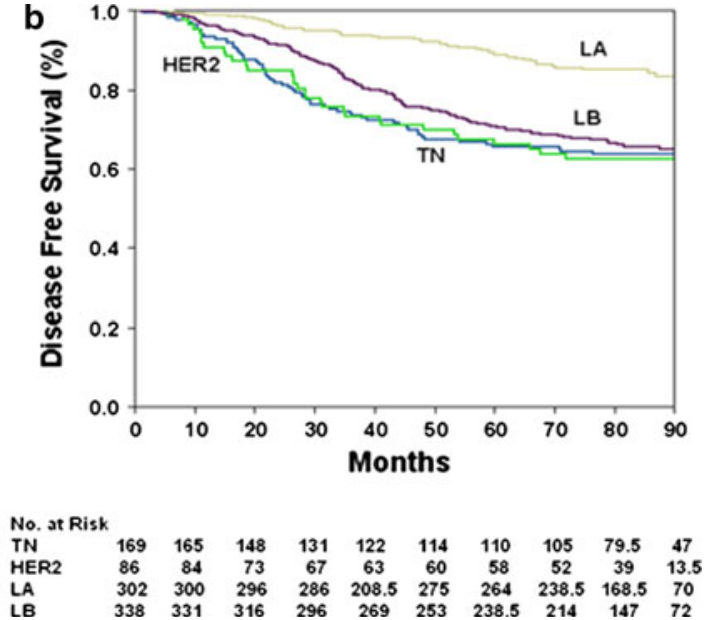

Fig. 2 DFS according to the two classifications. a hormone receptor/HER2 classification. b intrinsic subtypes (see text)

Figure 3 shows the DFS curves of the four HR/HER2 subgroups segregated with respect to the assigned treatment. FEC-P was statistically significantly superior to FEC only in the HR-/HER2 - subgroup. In the intrinsic subtype classification, FEC-P was statistically significantly superior to FEC in the TN and Luminal A subsets (Fig. 4). Since patients had not been stratified according to subtypes, a Cox's regression analysis of DFS was performed to rule out false positive results. The confounding variables included age, menopausal status, tumor size, histologic grade, histologic type, extra-capsular ganglionar invasion, number of lymph nodes, chemotherapy arm, and hormonal therapy. In the TN subgroup, menopausal status $(P=0.048)$, number of axillary lymph nodes involved $(P=0.049)$, and chemotherapy arm $(P=0.035)$ were independent variables predictive of DFS. In the Luminal A subgroup, histologic grade $(P=0.020)$, number of axillary lymph nodes involved $(P<0.0001)$, hormonal adjuvant therapy $(P=$ $0.001)$ and chemotherapy arm $(P=0.011)$ were independent variables predictive of DFS.

In the small subset of patients showing basal phenotype (TN and EGFR positive and/or cytokeratins $5 / 6$ positive; $n=79$ ), FEC-P was significantly superior to FEC, with 7-year DFS of 83 versus 57\% $(P=0.018)$ (Fig. 5). In this subgroup, the hazards ratio for DFS was 0.33 (95\%CI: $0.12-0.87 ; P=0.024)$.

\section{Discussion}

The 7-year follow-up update of GEICAM 9906 trial confirms a statistically significant advantage of FEC-P over FEC in terms of DFS (75 vs. $68 \%$ ) and shows now a significant benefit in OS (84 vs. $79 \%$ ) not seen in the 5-year 

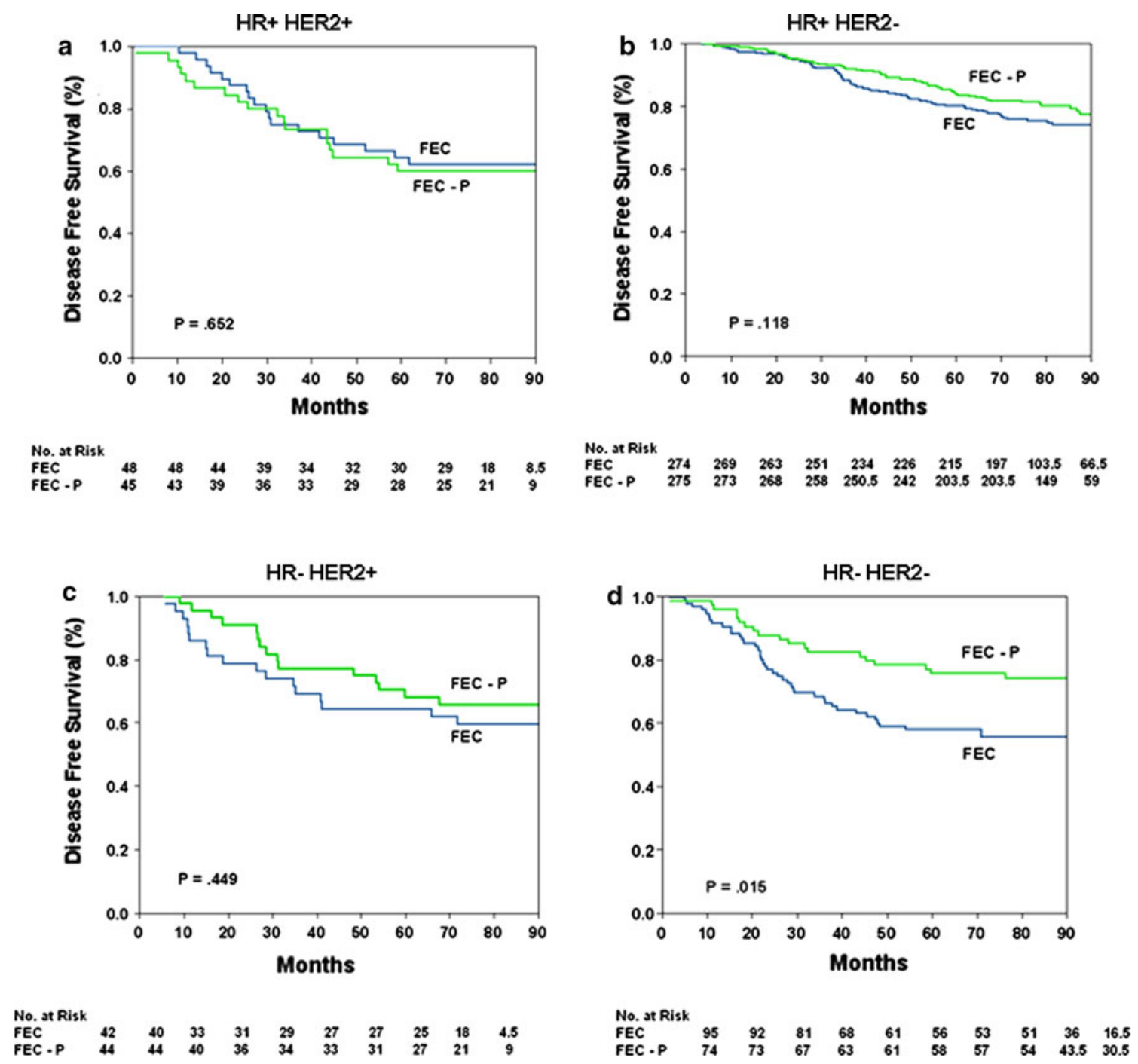

Fig. 3 DFS with FEC and FEC-P in the four subgroups of hormone receptor/HER2 classification (see text)

follow-up. The benefit with FEC-P is, however, small in absolute terms (7\% in recurrences and 5\% in deaths). Hence, using standard IHC and FISH methods to characterize patients, we attempted to define the subgroup of patients who do actually benefit from paclitaxel. The HR/ HER2 classification as well as the intrinsic subtype classification defined groups with good outcomes with chemotherapy with/without additional radiation and hormonal therapy (i.e., the HR+/HER2 - and Luminal A, with 7-year DFS of 77 and 85\%, respectively). Similarly, both classifications were coincident in showing poor prognosis for HR-/HER2+, and TN patients (7-year DFS of around $63 \%)$.

The DFS curves of the four intrinsic subtypes in the GEICAM 9906 are very similar to those of the same subgroup classification in the BCIRG 001 trial [10]. Since the advent of trastuzumab which significantly improves the treatment outcome in HER2+ patients [18, 19], the prognostic value of these two classifications has become reduced, with only the data of Luminal HER2 - and TN patients being of interest today.

The outcomes of the four subgroups (in terms of 3-year DFS) were somewhat similar in both trials: Luminal A (94\% GEICAM 9906 vs. 91\% BCIRG 001), Luminal B (82 vs. $82 \%)$, TN (75 vs. $67 \%$ ), and HER2 (73 vs. $68 \%$ ).

Using IHC/FISH classifications, we also sought to identify the subgroup of patients who would most benefit from the additional weekly paclitaxel. Overall, the maximum benefit with FEC-P was seen in the subset of TN patients. In these patients, FEC-P reduced the likelihood of relapse by $47 \%$, and yielded an absolute benefit in 7-year DFS of $18 \%$ with respect to FEC (74 vs. 56\%). A Cox regression indicated that treatment with FEC-P remained a significant predictor of DFS.

Of considerable note was that in the small subgroup of TN patients showing basal phenotype in our study, FEC-P 

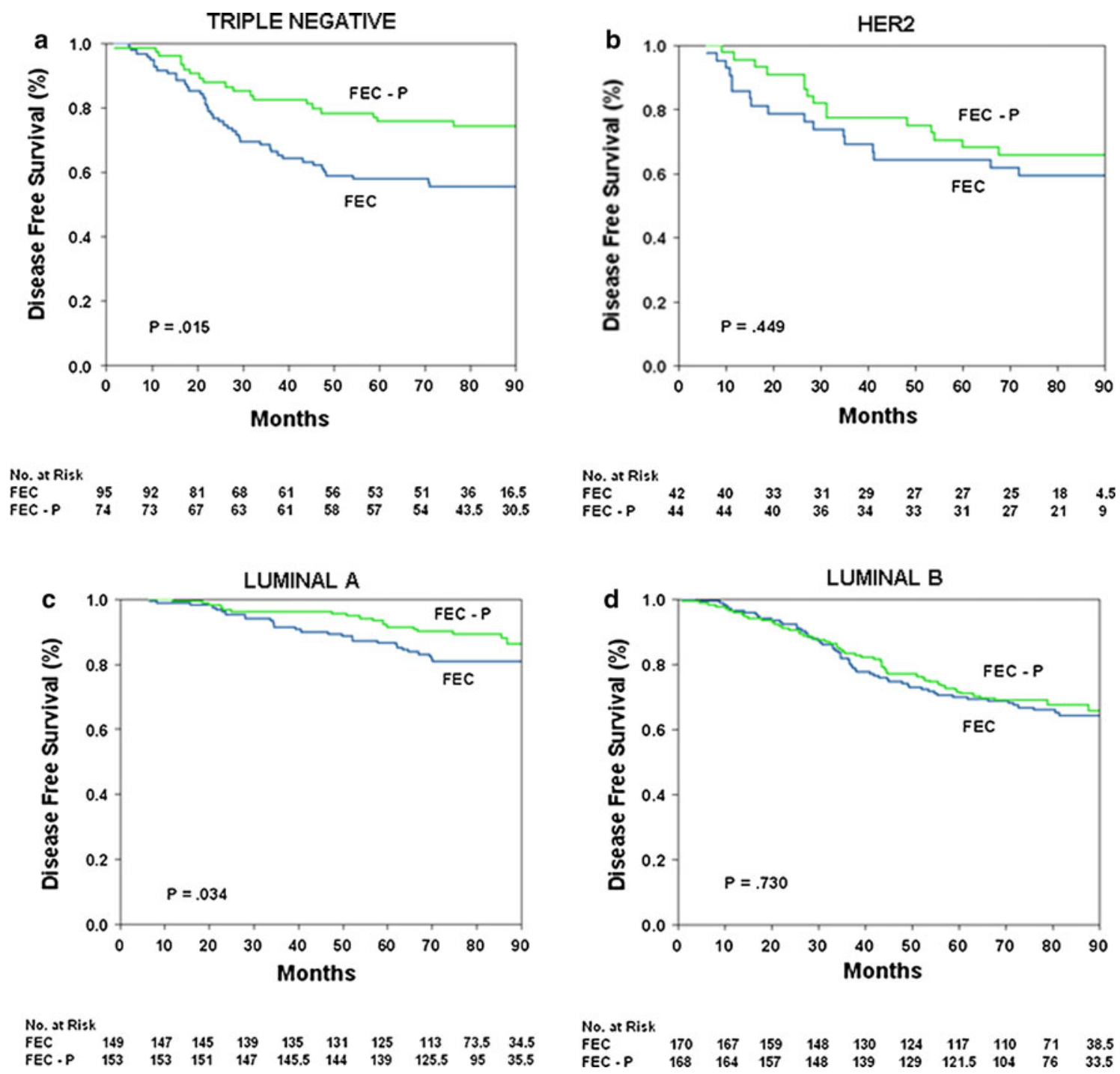

Fig. 4 DFS with FEC and FEC-P in the four subgroups of intrinsic subtype classification (see text)

treatment reduced the likelihood of recurrence by $67 \%$ compared to the treatment with FEC, and this difference was statistically significantly $(P=0.018)$ despite the small number of patients in this subgroup. The absolute benefit with FEC-P in terms of 7-year DFS was as high as $26 \%$. Again, a Cox regression analysis showed that treatment with FEC-P was an independent predictor of DFS. The poor outcome in the TN subgroup seen in our study with the standard FEC treatment (7-year DFS of 56\%) is similar to that reported by Tan et al. [20]. in a similar patient population treated with conventional adjuvant anthracyclines (7-year DFS of 58\%). The role of anthracyclinecontaining combinations as adjuvant therapy of TN tumors is currently being debated since anthracylines seem to be more efficacious than CMF-like regimens only in HER2 overexpressing tumors [21]. A recent analysis of the Canadian MA5 adjuvant trial in which, overall, six cycles of CEF were superior to six cycles of CMF [22], showed that patients with basal tumors (defined by IHC methods, as in our study) had a significantly better 5-year OS with $\mathrm{CMF}$ than with CEF (71 vs $51 \%$, respectively, $P=0.009$ ). The poor outcomes of TN patients with full dose epirubicincontaining combination in all these studies, also seen in the GEICAM 9906 trial, speaks against the use of anthracyclines in TN tumors.

Hayes et al. [8] published their results of the interaction of her2, paclitaxel treatment and outcome in the CALBG 9344 trial which compared four cycles of AC to AC followed by four cycles of paclitaxel. They observed that her2-positivity, regardless of estrogen receptor status, was associated with a significant benefit from paclitaxel administration (HR for recurrence of $0.59 ; P=0.01$ ). An exploratory analysis of the benefit of paclitaxel based on her2-positivity and estrogen receptor status was also 


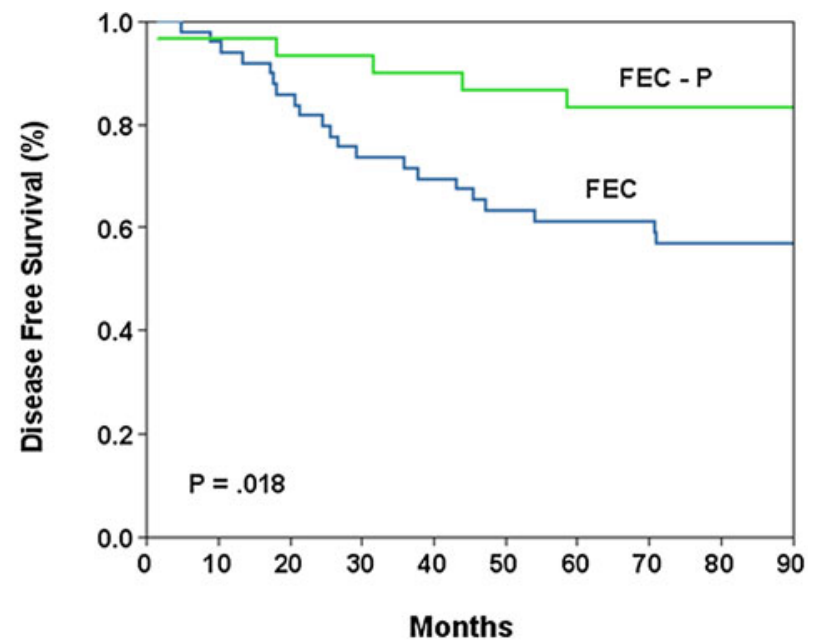

$\begin{array}{lllllllllll}\text { No. at Risk } & & & & & & & & & & \\ \text { FEC } & 49 & 48 & 42 & 37 & 34 & 31 & 29 & 28 & 20 & 8.5 \\ \text { FEC -P } & 30 & 29 & 28 & 28 & 27 & 26 & 25 & 25 & 19.5 & 14\end{array}$

Fig. 5 DFS with FEC and FEC-P in the subgroup of basal-like patients (triple negative plus either cytokeratine 5/6 positive or EGFR positive tumors)

performed, as in our study. In terms of DFS, the benefit of adding paclitaxel to AC was statistically significant in $\mathrm{HR}-/ \mathrm{HER} 2+$ patients $(P=0.001)$ and $\mathrm{HR}-/ \mathrm{HER}-$ patients $(P=0.002)$. HR $+/$ HER $2+$ obtained a benefit with paclitaxel of borderline significance $(P=0.058)$. The remaining patients (HR+/HER2-), appeared not to benefit from the addition of paclitaxel to AC. Some of the results of the CALGB 9344 trial have not been confirmed by the GEICAM 9906 trial. In our study, FEC-P was not statistically superior to FEC in the subgroups HR+/HER2-, $\mathrm{HR}-/ \mathrm{HER} 2+$ and HR+/HER2 + in the HR/HER2 classification. As in the CALGB 9344 trial, our study found a statistically significant benefit with paclitaxel in patients with HR - /HER - tumors, but the benefit of the addition of paclitaxel in the her2 positive subgroups observed in the CALGB 9344 trial was not clear in our trial. The differences in subtype analyses seen in these two trials could be due to several factors. First, there was an inequality in the CALGB 9344 trial in terms of duration of treatment (four vs. eight cycles); thus the benefit seen could have been due merely to a more protracted chemotherapy regimen rather than a taxane-specific effect. Second, the control arm (four cycles of AC) could be a suboptimal adjuvant treatment regimen, particularly in patients whose tumors overexpress her2. Since her2-positive patients obtain the maximum benefit with anthracyclines [21], the administration of an appropriate anthracycline regimen is of the critical importance for this particular subset of patients. Finally, the differences in subset results of the above-mentioned trials could be due to the reduced study's power to detect statistically significant treatment differences in those subsets with small number of patients.

In our study, we found an unexpected superiority of FEC-P over FEC in the Luminal A subtype, confirmed in a multivariate analysis that included other relevant variables such as number of axillary lymph nodes involved, tumor grade, menopausal status and hormonal therapy. In the BCIRG 001 trial TAC was not superior to FAC in the Luminal A subset. Similarly, in the French PASC 01 trial, Luminal A patients (hormone receptor positive patients with low Ki 67 expression), apparently did not get any benefit from the addition of sequential docetaxel to FEC $[23,24]$. The reasons for this discrepancy remain unclear.

In the BCIRG 001, TAC was associated with a better DFS than FAC in the HER2 subset; albeit the differences did not reach statistical significance $(P=0.068)$ probably due to the sample size $(n=113)$. In the GEICAM 9906 trial, no conclusion can be drawn in this subset due to the same reason (only 86 patients in the HER2 subtype). BCIRG 001 and GEICAM 9906 were coincident in showing an advantage of the taxane arm in the TN patient subpopulation although, in the BCIRG trial, the difference was of borderline significance $(P=0.051)$. A a subgroup analysis of the French PACS 01 trial (comparing six cycles of FE100C with three cycles of FE100C followed by three cycles of docetaxel) found that the benefit with docetaxel was mainly confined to the basal-like subtype [23, 24]. The activity of taxanes in TN/basal tumors, seen in all these retrospective analyses, would be particularly relevant since no targeted therapy is currently available for this patient population.

Retrospective subset analyses intended to identify subsets of patients who obtain the maximum benefit from a particular therapy, are now common in adjuvant breast cancer trials. However, the interpretation of results requires caution. For example, multiple, unplanned analyses can lead to spurious treatment correlations (false positive results). Further, the post-hoc segregation of the overall patient population into subsets reduces the study's power to detect statistically significant treatment differences, since many of the subsets become very reduced in size leading to potential false negative results. Finally, there is a real possibility of publication bias, i.e., negative results (lack of association) are seldom submitted for publication by the investigators and, when submitted, are rarely accepted by medical journals. Because of all these caveats, the results of such subset analyses are considered merely hypothesis generating.

Unfortunately, in real life, the majority of the interesting hypotheses generated by retrospective analyses will never be tested in prospective trials. For instance, a prospective trial intending to confirm the lack of superiority of TAC over FAC in Luminal A patients, will probably never be 
conducted because of ethical concerns, difficulties in enrolment, together with the protracted trial period and follow-up needed to generate conclusive results. Therefore, a renewed scientific debate on the real value and practical applications of retrospective subset analysis seems increasingly necessary.

In summary, the IHC subgroup analyses of GEICAM 9906 trial, although retrospective and unpowered for some subsets, firmly suggest that the benefit from FEC-P over FEC in 7-year DFS is mainly due to the effect of paclitaxel in the TN/basal phenotypes, an observation common to other taxane studies. The potential benefit seen with paclitaxel in Luminal A patients is intriguing and need to be confirmed in other trials.

Acknowledgments The authors thank Maribel Casas, and María José Escudero (GEICAM) for their assistance in preparing the manuscript. Editorial help was by Peter R. Turner of t-SciMed (Reus, Spain). This work was supported, in part, by grants from BristolMyers Squibb and Pharmacia.

\section{Appendix}

The following investigators, with their affiliations, participated in the GEICAM 9906 trial:

Hospital General Universitario Gregorio Marañón, Madrid, Spain-M. Martín and J. A. LópezGarcía-Asenjo; Hospital Universitario de Elche, Elche, Spain-Á. RodríguezLescure; Instituto Valenciano de Oncología, Valencia, Spain-A. Ruiz and S.Almenar; Hospital Virgen de la Victoria, Málaga, Spain-E. Alba and L. Vicioso;Complexo Hospitalario Universitario A Coruña, La Coruña, SpainL. Calvo and M. D. Guitián; Hospital Vírgen del Rocío, Sevilla, Spain-M. Ruiz-Borrego and J. Palacios; Hospital $\mathrm{La} \mathrm{Fe}$, Valencia, Spain-B. Munárriz and F. Vera; Hospital Universitario de Salamanca, Salamanca, Spain-C. A. Rodríguez; Hospital Ramón y Cajal, Madrid, Spain-C. Crespo and J. F. González-Palacios; Centro Oncológico de Galicia, La Coruña, Spain-M. Ramos and A. de la Cruz; Hospital de Cabueñes, Gijón, Spain-J. M. Gracia and J. Martín; Hospital Clínico de Valencia, Valencia, SpainA. Lluch and J. Ferrer; Hospital Donostia, San Sebastián, Spain-I. Álvarez and I. Ruiz; Hospital Parc Taulí, Sabadell, Spain-M. Á. Seguí and A. Sáez; Hospital Clínico Lozano Blesa, Zaragoza, Spain-J. I. Mayordomo and M. Moros; Hospital Miguel Servet, Zaragoza, Spain-A. Antón and M. José Ríos; Complejo Hospitalario Puerta del Mar, Cádiz, Spain—J. M. Baena and M. J. Palomo; Instituto Oncológico de Guipúzcoa, San Sebastián, Spain-A. Plazaola and R. Rezola; Clínica Corachan, Barcelona, Spain-A. Modolell and P. de las Heras; Hospital Universitario San Joan de Reus, Reus, Spain-A. Pelegrí and F. Riu; Complejo Hospitalario Xeral Calde, Lugo, Spain-J. R. Mel and
J. Alba; Hospital Provincial de Córdoba, Córdoba, SpainE. Aranda and E. Fuentes; Hospital General de Alicante, Alicante, Spain-E. Adrover and G. Peiró; Hospital Provincial de Zamora, Zamora, Spain-J. V. Álvarez and J. C. Solera; Hospital San Cecilio de Granada, Granada, SpainJ. L. G. Puche and J. Aneiros; Hospital Ciudad de Jaén, Jaén, Spain-P. Sánchez-Rovira and C. Cueva; Hospital Mutua de Terrasa, Terrasa, Spain-S. Gonzalez and A. Salas; Hospital Marqués de Valdecilla, Santander, Spain-J. M. LópezVega and M. Francisca Garijo; Hospital Txagorritxu, Vitoria, Spain-S. Domínguez and $\mathrm{M}^{\mathrm{a}}$. A. Viguri; Hospital Comarcal Barbastro, Barbastro, Spain-J. Florian and R. Oncins; Hospital Universitario De Canarias, Tenerife, Spain-N. Batista and R. Méndez; Hospital de Elda, Elda, Spain-C. Llorca and S. Alonso Hernández; Hospital General de Móstoles, Móstoles, Spain-M. Méndez and M. J. Mestre; Hospital Clínico Barcelona, Barcelona, SpainM. Muñoz and P. Fernández; Hospital General Universitario de Valencia, Valencia, Spain-Ma $\mathbf{M}^{\mathrm{a}}$ J. Godes and A. Pérez; Hospital General de Ciudad Real, Ciudad Real, Spain-M. J. García and M. Carbajo; Hospital Puerta de Hierro, Madrid, Spain-R. Cubedo and E. Tejerina; Althaia Xarxa Assistencial Manresa, Manresa, Spain-I. Guash and L. Sardans; Hospital General de Jerez, Jerez, Spain-A. Moreno and A. Rodríguez; Hospital Universitario Insular, Las Palmas, Spain-A. Murias and M. Pavcovich; Fundación Hospital de Alcorcón, Alcorcón, Spain-C. Jara and M.Argüelles; Hospital Juan Ramón Jiménez, Huelva, Spain-B. Calero and J.Conde; Hospital Arnau de Vilanova, Lérida, Spain-V. Alberola and J. Fernando;Hospital General Universitario de Guadalajara, Guadalajara, Spain-J. Casinello and J. Cuevas; Hospital Municipal de Badalona, Badalona, Spain-I. Moreno and M. Gómez; Hospital de Cruces, Barakaldo, Spain-J. R. Barceló and J. $\mathrm{M}^{\mathrm{a}}$ Ribera; Hospital del Mar, Barcelona, Spain-I. Tusquets and J. $\mathrm{M}^{\mathrm{a}}$ Corominas; Hospital de León, León, Spain-A. García and T. Arribas; Complejo Hospitalario de Orense, Orense, Spain-J. García and C. Peñin; Hospital Río Carrión, Palencia, Spain-A. Arizcum and L. Pérez; Hospital Universitario de Gran Canaria Doctor Negrín, Las Palmas, SpainJ. Aguiar and M. A. Limeres; Hospital Virgen de la Salud, Toledo, Spain-M. A. de la Cruz and J. Martín; Hospital de L'Esperit Sant, Santa Coloma de Gramenet, Spain-C. Martín and $\mathrm{M}^{\mathrm{a}}$ A. Ruiz; Hospital de la Ribera, Valencia, J. Miguel and R. Cano; Hospital de Navarra, Pamplona, Spain; J. J. Valerdi and A. Córdoba; Hospital Universitario de Valladolid, Valladolid, Spain-F. López and I. Peral; Hospital Morales Messeguer, Murcia, Spain-F. Ayala and M. A. Chaves; Hospital Germans Trias y Pujol, Badalona, Spain-A. Barnadas and E. Castellá; Hospital Virgen de los Lírios, Alicante, Spain-A. Oltra and J. L. Losa; Hospital Universitario Puerto Real, Puerto Real, Spain-A. Lorenzo and A. Hens; Hospital Doctor Peset, Valencia, Spain-D. 
Almenar and J. Escandón; Hospital Consorci Sanitari Terrasa, Terrassa, Spain-A. Arcusa and I. Roig; Hospital Puerto de Sagunto, Valencia, Spain-A. Galán and B. Martínez; Hospital de la Arrixaca, Murcia, Spain-J. L. Alonso and J. Bermejo; Hospital Provincial de Toledo, Toledo, Spain-P. Puñal and C. Pérez.

\section{References}

1. Henderson IC, Berry DA, Demetri GD et al (2003) Improved outcomes from adding sequential paclitaxel but not from escalating doxorubicin dose in an adjuvant chemotherapy regimen for patients with node-positive primary breast cancer. J Clin Oncol 21:976-983

2. Mamounas EP, Bryant J, Lembersky BC et al (2005) Paclitaxel after doxorubicin plus cyclophosphamide as adjuvant chemotherapy for nodepositive breast cancer: results from NSABP-B 28. J Clin Oncol 23:3686-3696

3. Martin M, Pienkowski T, Mackey J et al (2005) Adjuvant docetaxel for node positive breast cancer. $\mathrm{N}$ Engl $\mathrm{J}$ Med 352:2302-2313

4. Roche H, Fumoleau P, Spielmann M et al (2006) Sequential adjuvant epirubicin-based and docetaxel chemotherapy for nodepositive breast cancer patients: the FNCLCC PASC 01 Trial. J Clin Oncol 24:5664-5671

5. Jones SE, Savin MA, Holmes FA et al (2006) Phase iii trial comparing doxorubicin plus cyclophosphamide with docetaxel plus cyclophosphamide as adjuvant therapy for operable breast cancer. J Clin Oncol 24:5381-5387

6. Jones S, Holmes FA, O'Shaughnessy JO et al (2009) Docetaxel with cyclophosphamide is associated with an overall survival benefit compared with doxorubicin and cyclophosphamide: 7-year follow-up of US oncology research trial 9735. J Clin Oncol 27:1177-1183

7. Martin M (2006) Molecular biology of breast cancer. Clin Trasl Oncol 8:7-14

8. Hayes DF, Thor AD, Dressler LG et al (2007) HER2 and response to paclitaxel in node-positive breast cancer. N Engl J Med 357:1496-1506

9. Parker JS, Mullins M, Cheang MC et al (2009) Supervised risk predictor of breast cancer based on intrinsic subtypes. J Clin Oncol 27(8):1160-1167

10. Hugh J, Hanson J, Cheang MCU et al (2009) Breast cancer subtypes and response to docetaxel in node-positive breast cancer: use of an immunohistochemical definition in the BCIRG 001 trial. J Clin Oncol 27:1168-1176

11. Martin M, Rodriguez-Lescure A, Ruiz A et al (2008) Randomized phase 3 trial of fluorouracil, epirubicin, and cyclophosphamide alone or followed by paclitaxel for early breast cancer. $\mathbf{J}$ Natl Cancer Inst 100:805-814

12. Hudis CA, Barlow WE, Costantino JP et al (2007) Proposal for standardized definitions for efficacy end points in adjuvant breast cancer trials: the STEEP system. J Clin Oncol 25:2127-2132

13. Allred DC, Harvey JM, Berardo M, Clark GM (1998) Prognostic and predictive factors in breast cancer by immunohistochemical analysis. Mod Pathol 11:155-168

14. Sauter G, Lee J, Barlett JMS, Slamon DJ, Press MF (2009) Guidelines for human epidermal growth factor receptor 2 testing: biological and methodological considerations. J Clin Oncol 27:1323-1333

15. Cheang MCU, Voduc D, Badjik C et al (2008) Basal-like breast cancer defined by five biomarkers has superior prognostic value than triple-negative phenotype. Clin Cancer Res 14:1368-1376

16. McShane LM, Altman DG, Sauerbrei W et al (2005) Reporting recommendations for tumor marker prognostic studies. J Clin Oncol 23:9067-9072

17. Kaplan EL, Meier P (1958) Nonparametric estimation from incomplete observations. J Am Stat Assoc 53:457-481

18. Piccart-Gebhart M, Procter M, Leyland-Jones B et al (2005) Trastuzumab after adjuvant chemotherapy in HER2-positive breast cancer. N Engl J Med 353:1659-1672

19. Romond EH, Perez EA, Bryant J et al (2005) Trastuzumab plus adjuvant chemotherapy for operable her2-positive breast cancer. N Engl J Med 353:1673-1684

20. Tan DSP, Marchio C, Jones RL et al (2007) Triple negative breast cancer: molecular profiling and prognostic impact in adjuvant anthracyline-treated patients. Breast Cancer Res Treat 111:27-44

21. Gennari A, Sormani MP, Pronzato P et al (2008) HER2 status and efficacy of adjuvant anthracyclines in early breast cancer: a pooled analysis of randomized trials. J Natl Cancer Inst 100:14-20

22. Cheang M, Chia SK, Tu D et al (2009) Anthracyclines in basal breast cancer: the NCI-CTG trial MA5 comparing adjuvant CMF to CEF. J Clin Oncol 27(15s):519

23. Penault-Llorca F, André F, Sagan C et al (2009) Ki 67 expression and docetaxel efficacy in patients with estrogen receptor-positive breast cancer. J Clin Oncol 27:2809-2815

24. Jacquemier J, Penault-Llorca F, Mnif $\mathrm{H}$ et al (2006) Identification of a basal-like subtype and comparative effect of epirubicin-based chemotherapy and sequential epirubicin followed by docetaxel chemotherapy in the PACS 01 breast cancer trial: 33 markers studied on tissue-microarrays (TMA). J Clin Oncol 24(18S):509 